302-2015.R3

Original Article

\title{
What is the likelihood of colorectal cancer when surgery for ulcerative colitis associated dysplasia is deferred?
}

Authors: Jamie Murphy PhD FRCS, ${ }^{1}$ Kristen A Kalkbrenner PA-C, ${ }^{2}$ Joseph V Blas MD, ${ }^{2}$ John H Pemberton MD, ${ }^{3}$ Ron G Landmann MD, ${ }^{4}$ Tonia M Young-Fadok MD MS, ${ }^{2}$ David A Etzioni MD MSHS. ${ }^{2}$

\section{Institutions:}

1: Department of Surgery \& Cancer, St. Mary's Hospital, Imperial College London, UK.

2: Division of Colon and Rectal Surgery, Mayo Clinic, Scottsdale, AZ USA.

3: Division of Colon and Rectal Surgery, Mayo Clinic, Rochester, Minnesota, USA.

4: Division of Colon and Rectal Surgery, Mayo Clinic, Jacksonville, Florida, USA.

Corresponding Author: Mr. Jamie Murphy, Senior Lecturer in Colorectal Surgery, St. Mary's Hospital, Imperial College London, London, UK.

Telephone: 4805129174

Fax: $480-342-2866$

E-mail: jamie.murphy@doctors.net.uk 
Conflict of Interest: None

Word Count: $\quad$ Abstract: 244
Category Submitted: Original article

Text: 2,530

Authorship Credits: JM: Data management, data analysis and interpretation, drafting and final approval of manuscript; KAK: Data management, data analysis and interpretation, revision and final approval of manuscript; JVB: Data management, data analysis and interpretation, revision and final approval of manuscript; JHP: Data interpretation, revision and final approval of manuscript; RGL: Data interpretation, revision and final approval of manuscript; TMYF: Data interpretation, revision and final approval of manuscript; DAE: Data analysis, data interpretation, revision and final approval of manuscript. 


\section{ABSTRACT}

Aim: Surgery aims to prevent cancer-related morbidity for patients with ulcerative colitis (UC)-associated dysplasia. The literature varies widely regarding the likelihood of dysplastic progression to higher grades of dysplasia or cancer. The aim of this study was to characterise the likelihood of the development of colorectal cancer (CRC) of patients with UC-associated dysplasia who chose to defer surgery.

Method: A retrospective review was carried out of patients undergoing surgery for UC at the Mayo Clinic, who were diagnosed to have dysplasia between August 1993 and July 2012. The relationships between grade of dysplasia, time to surgery and the detection of unsuspected carcinoma were investigated.

Results: 175 patients underwent surgery at a median of 4.9 [IQR:2.5-8.9] months) after a diagnosis of dysplasia. Their median age was 52 (IQR:43-59) years. An initial diagnosis of indeterminate dysplasia was not associated with CRC (0/23; 17.7[8.1-29.6] months). Thirty six patients who had an initial diagnosis of dysplasia progressed from indeterminate to low-grade dysplasia (24.2[11.0-30.4] months). Low-grade dysplasia was associated with a $2 \%(1 / 56$; T2N0M0) risk of CRC when present in random surveillance biopsies and a $3 \%(2 / 61$; T1N0M0, T4N0M0) risk if detected in endoscopically visible lesions (7.4[5.2-33.3] months). Eighteen patients progressed from indeterminate to highgrade dysplasia (19.1 [9.2-133.9] months). Seventeen patients progressed from low- to high-grade dysplasia (11.0[5.8-30.1] months). None of the patients with high-grade dysplasia (0/35) progressed to CRC (4.5[1.7-9.9] months).

Conclusion: Dysplasia was associated with a low incidence of node negative CRC if surgery was deferred for up to five years. These findings may help inform the decisionmaking process for asymptomatic patients who are having to decide between intensive surveillance or surgery for UC associated-dysplasia. 


\section{What does this paper add to the literature?}

The study demonstrates UC-associated dysplasia is associated with a small likelihood of node negative cancer if surgery is deferred for up to five years. These findings may assist the decision-making process for patients with asymptomatic UC who are considering intensive surveillance rather than surgery after a diagnosis of dysplasia. 


\section{INTRODUCTION}

Ulcerative colitis (UC) is associated with a risk of colorectal cancer (CRC) that increases with the duration of the disease ${ }^{1-3}$ Genetic mutations associated with CRC arising in inflamed bowel are thought to differ from sporadic cancer pathways, but dysplasia has been firmly identified as the precursor of carcinoma. Endoscopic surveillance protocols have been developed to detect dysplasia at an early stage. The extent of dysplastic change within random / targeted biopsies or polypectomy specimens is an important biomarker when stratifying the CRC risk for individual patients ${ }^{4}$ with the interval between endoscopic assessments tailored to the extent and duration of the disease, in addition to the grade of dysplasia identified. ${ }^{5,6}$

Systematic review of published data estimates that low-grade dysplasia in UC may confer a nine fold life time risk of developing cancer and a twelve fold life time risk of developing high-grade dysplasia or CRC. ${ }^{7}$ There is considerable variation among studies with reports of progression of low-grade dysplasia to invasive cancer in $0-57 \%$ of cases ${ }^{8-}$ ${ }^{12}$. In the light of this, national guidelines err on the side of prophylactic surgery even for asymptomatic patients as the risk of CRC is so poorly characterised. ${ }^{5,6,13}$ This is despite the significant morbidity of major surgery in addition to the risk of a permanent stoma and poor quality of life should restorative procedures fail. Surgery may be considered overtreatment given data that suggest that the rate of progression from dysplasia to cancer may be as low as $5 \%$ at four years. ${ }^{8}$ Moreover there are now surveillance programmes aimed to detect and treat dysplasia or early cancer by endoscopy, even for patients with chronic, extensive UC. ${ }^{14}$ Patients may have great difficulty in deciding whether to 
proceed with surgery for dysplasia after an informed discussion, especially if the symptoms of UC are well controlled. ${ }^{15}$

The aim of the present study was to determine the risk of developing a colorectal cancer when surgery for UC-associated dysplasia is deferred. Such an analysis should inform the clinical decision-making process and guide patients on how long surgery may safely be deferred when they are diagnosed with dysplasia. 


\section{METHOD}

\section{$\underline{\text { Inclusion criteria }}$}

The study was approved by the Mayo Clinic institutional review board. The patient cohort included in this study was established from the billing records of the three academic hospitals that constitute the Mayo Clinic (Jacksonville FL, Rochester MN and, Scottsdale AZ). Patients who underwent total abdominal colectomy, restorative proctocolectomy or proctocolectomy with end stoma construction between August 1993 and July 2012 were identified using an institutional discharge database by searching for International Classification of Diseases, Ninth Revision, Clinical Modification [ICD-9$\mathrm{CM}]$ ) procedural codes (codes $45.7 \mathrm{x}, 45.8)$. The cohort was restricted to include only those patients with an ICD-9-CM code pertinent to UC (556.xx).

\section{$\underline{\text { Exclusion Criteria }}$}

Distinct exclusion criteria were applied to make the studied cohort as homogenous as possible. Based on a review of the electronic medical record and cancer registry data, the following exclusion criteria were stipulated: a pre-operative histopathological diagnosis of UC-associated invasive cancer at index colonoscopy, a known or suspected polyposis syndrome, an indication for surgery other than UC (including familial adenomatous polyposis, Crohn's, or diverticular disease), pre-operative endoscopy reports that were unavailable, an interval between initial diagnosis of dysplasia and surgery of less than three months, as this time frame was arbitrarily deemed as a logistic rather than an intentional delay and, no identifiable pre-operative pathology report. 


\section{$\underline{\text { Data Abstraction }}$}

The medical charts of patients with UC who did not undergo surgery for three months or more were reviewed to determine the association between time to surgery and dysplastic progression / development of CRC. Endoscopic, operative, and histopathological reports were abstracted to collect the following data: patient factors (age, sex, disease duration, family history of colorectal cancer, primary sclerosing cholangitis), the presence/absence of endoscopically visible lesions, the interval between pre-operative biopsies identified as containing indeterminate/low-/high- grade dysplasia, the surgical procedure undertaken, the indication for surgery and the post-operative histological identification of cancer. In cases where more than one grade of dysplasia was identified, the highest grade was considered as the index area of dysplasia and all data were abstracted based on that lesion. Endoscopy reports were found to be highly inconsistent with regard to characterising the morphology of visible lesions harbouring dysplasia (e.g adenomatous polypoid lesions or dysplasia associated lesion or mass [DALM]). These data were therefore abstracted as one pooled group and designated 'endoscopically visible lesions'.

\section{$\underline{\text { Statistical Analyses }}$}

Data analyses were performed using statistical analysis software (SAS, Cary, NC, USA). Non-parametric data were given by median and inter-quartile range values. Hazard ratios with $95 \%$ confidence limits $[\mathrm{CI}]$ were computed using the progression of indeterminate to low-grade dysplasia as the comparator group 


\section{RESULTS}

\section{$\underline{\text { Patient demographics }}$}

The study included 175 patients who had undergone surgery a minimum of three months after a pre-operative diagnosis of dysplasia with a median time to surgery of 4.9 (interquartile range [IQR]: 2.5-8.9]; mean 22.1+/-30.9) months. The median patient age was 52 (IQR: 43-59) years for the studied cohort, with 101 (58\%) patients younger than 55 years, $43(26 \%)$ aged 55 to 64 years, $25(14 \%) 65$ to 74 years and six (2\%) over 74 years. There were 124 males and 51 females (71\% vs. 29\%). Eighty nine (51\%) underwent surgery for dysplasia, while the remainder chose surgical intervention for symptom control. Figure 1 outlines the distribution of cases by year during the study period. The reported median pre-operative duration of colitis in the study group was 12.5 (IQR: 7-20) years. A significant proportion of patients reported a family history of colorectal cancer $(\mathrm{n}=23 ; 13 \%)$ or had previously been diagnosed with primary sclerosing cholangitis ( $\mathrm{n}=35 ; 20 \%)$. Eighty (46\%) patients had multifocal dysplasia.

To exclude the possibility of selection bias the study cohort was compared with patients at the Mayo Clinic who had been diagnosed with UC-associated dysplasia but who chose to undergo immediate surgery $(\mathrm{n}=154)$. No statistically significant difference in demographics, disease duration or previous personal or family medical history was identified when comparing these two groups. 


\section{$\underline{\text { Surgery }}$}

Restorative proctocolectomy was the most commonly performed surgical procedure in this patient cohort $(\mathrm{n}=109$ [62\%]). The majority of the remaining patients either underwent proctocolectomy with end ileostomy construction $(n=47[27 \%])$ or total colectomy and end ileostomy formation ( $\mathrm{n}=9$ [5\%]). Subtotal colectomy and restoration of gastrointestinal continuity was also performed for a small number of patients $(\mathrm{n}=10$ [6\%]) having an ileorectal (9) or ileosigmoid anastomosis (1).

$\underline{\text { Time to surgery and histopathology results }}$

\section{Cohort as a whole}

The index grade of dysplasia and progression of dysplastic change for the group overall are shown in Figure 2.

\section{Indeterminate dysplasia}

A total of 23 patients identified with preoperative indeterminate dysplasia had surgical intervention after a median of 17.7 (IQR: 8.1-29.6) months (Figure 3). During endoscopic assessment no patients were identified as having endoscopically visible dysplastic lesions. Ten patients had multi-focal indeterminate dysplasia. The majority of patients underwent surgery for failed medical therapy; however, in six cases between 1997-1999 the presence of indeterminate dysplasia was considered an indication for surgery. All surgical specimens were negative for cancer. The risk of indeterminate dysplasia progressing over the study period is outlined in Figure 4. 


\section{Low-grade dysplasia}

Similarly, 117 patients identified as having preoperative low-grade dysplasia underwent surgical resection after a median delay of 10.9 (IQR: 5.2-22.1) months (Figure 3). Thirtysix (31\%; median 24.2 (IQR: 11.0-30.4) months) patients were identified to have progressed from indeterminate dysplasia. During endoscopic assessment 61 patients were identified as having endoscopically visible dysplastic lesions. Forty-nine patients were identified as having multi-focal low-grade dysplasia. In addition, three patients were identified as having developed CRC during follow up (hazard ratio [HR]: 1.332 [95\%C.I.: 1.119 to 1.509]; initial dysplasia diagnosis: 1 patient - screening biopsies; 2 patients endoscopically visible lesions). The first patient was identified at the 3-month follow-up period as having developed a $1.3 \mathrm{~cm} \mathrm{~T} 2 \mathrm{~N} 0 \mathrm{M} 0$ colon cancer on a background of multi-focal low-grade dysplastic change on screening biopsies alone. The second patient was identified at 7.4 months as having developed a $5 \mathrm{~cm}$ T4 N0 M0 colon tumour again following a prior diagnosis of multi-focal low-grade dysplasia. The third individual was determined to have a $1.4 \mathrm{~cm} \mathrm{T1} \mathrm{N0} \mathrm{M0} \mathrm{colon} \mathrm{cancer} \mathrm{at} 59.1$ months of follow-up (Figure 4). No patients identified as having preoperative low-grade dysplasia developed rectal cancer and all of the other surgical specimens $(n=114)$ were negative for malignancy in this group.

\section{High-grade dysplasia}

Finally, 35 patients identified as having pre-operative high-grade dysplasia underwent surgical intervention after a median delay of 4.5 (IQR: 1.7-9.9) months (Figure 3). Of this cohort, eighteen (51\%; median follow up - 19.1 (IQR: 9.2-133.9) months; HR: 0.803 
[95\%C.I.: -1.494 to 2.528$]$ ) patients were identified to have progressed from indeterminate dysplasia diagnosed on previous examinations (Figure 4). Similarly, seventeen (49\%; median follow up - 11.0 (IQR: 5.8-30.1) months; HR: 1.865 [95\%C.I.: 0.0809 to 2.1437 ) patients were identified who progressed from low- to high-grade dysplasia during the study period (Figure 4). Although 13/35 (37\%) patients were demonstrated to have endoscopically visible dysplastic lesions during follow up, no patients were identified as having a pre-operative diagnosis of cancer and all surgical specimens were negative for malignancy. 


\section{DISCUSSION}

The study has shown UC-associated dysplasia is associated with a small likelihood of node negative cancer if surgery is deferred for up to five years. Furthermore, the risk of dysplastic progression from indeterminate to low-, or low- to high-grade, dysplasia in this cohort was relatively small. These data deviate significantly from a number of previous studies and suggest that intensive surveillance may be appropriate for asymptomatic patients with UC-associated dysplasia who wish to defer surgery.

For this large cohort of 175 patients we identified a 2-3\% likelihood of developing CRC in the setting of histologically confirmed low-grade dysplasia over a median study period of 4.9 (IQR: $2.5-8.9$; mean 22.1+/-30.9) months. Two of the patients with cancer had previously been diagnosed with multi-focal low-grade dysplasia and in two cases the large size the lesion and the interval between identification of dysplasia and the diagnosis of malignancy, suggest that $\mathrm{CRC}$ was missed at the index endoscopic assessment rather than these being true interval cancers. If this assumption is correct the risk of developing CRC following a diagnosis of low-grade dysplasia was actually $0.6 \%$.

While six individuals in this study underwent surgery for indeterminate dysplasia alone, these procedures occurred in the 1990s when the risk of progression was less well understood. Eighteen patients progressed from indeterminate to high-grade dysplasia over a period of 19.1 (IQR: 9.2-133.9) months. A further seventeen progressed from low- to high-grade dysplasia over 11.0 (IQR: 5.8-30.1) months. None of the patients diagnosed with high-grade dysplasia were found to have CRC in the surgical resection specimen. 
This is not the first study to assess the progression of dysplasia in patients who choose to undergo surveillance rather than surgery. ${ }^{8,12,16,17}$ Consequently, it should be interpreted in the context of the existing published literature. The presence of low-grade dysplasia has previously been associated with a $1-69 \%$ risk of progression to CRC. ${ }^{8,12,16,17}$ One of the largest of these studies was reported in 2012 by Zisman et al ${ }^{8}$ which described dysplastic progression of low-grade lesions in 42 patients with dysplasia associated with UC. The incidence of dysplastic progression to CRC was $5 \%$, while high -grade evolved from low-grade dysplasia in $14 \%$ of patients.

Several previous studies report rates of progression of dysplasia leading to CRC substantially greater than that found in the present study although the reasons are unclear. The discrepancy may in part have been due to differences in the duration of follow up, but even studies ${ }^{8,12}$ with similar follow up intervals also report very different results highlighting the need to review the methodology of any study when the rate of development of CRC is being discussed. The data presented within this study are more in keeping with the work of Zisman et al who reported that the majority of UC patients with LGD did not progress to higher grades of dysplasia during a 4 year follow up. The shorter follow up period of our study may possibly explain the slightly lower rates of dysplastic progression and CRC found in our series. Nevertheless, further well-conducted prospective studies are necessary to confirm the findings presented above before surveillance of patients with UC associated dysplasia can be safely carried out as an alternative to surgery. Recent advances in endoscopic surveillance may also make the 
possibility of long-term surveillance more practical, particularly in the light of new techniques such as narrow-band imaging, advanced endoscopic chromoendoscopy, (auto)-fluorescence imaging, magnification colonoscopy with pit pattern analysis and confocal endomicroscopy becoming more popular. ${ }^{1,18-21}$

The study has strengths and limitations. It is a retrospective chart review, but it includes a large number of patients with prospectively recorded detailed endoscopic, surgical and histopathological data. As as retrospective study, it is however subject to bias. Furthermore the practice and population of patients attending the Mayo Clinic may not be representative of other patient groups, especially as $99 \%$ of pre-operative biopsies were re-reviewed by two specialised gastrointestinal pathologists. In addition, the inability of this study to accurately discriminate between a simple adenoma and a DALM (dysplasia associated lesion or mass) further complicates the presented data. This study also did not address the fate of individuals who were under surveillance for UC-associated dysplasia but who did not undergo surgery. Finally, our institution did not have a uniform protocol for endoscopic surveillance, sampling of areas adjacent to visible lesions or the treatment of raised dysplastic lesions. The contemporary standard of care would be to remove raised lesions fully wherever possible, biopsy the surrounding mucosa, document the endoscopic appearance of the associated lumen and submit all samples for histopathological assessment. Fully excised lesions with no surrounding dysplasia that had developed in macroscopically uninflamed bowel would be deemed to require no further treatment, with other scenarios resulting in a detailed discussion with the patient regarding the relative merits of surgery or surveillance. 
The present study has demonstrated that deferring surgery after a diagnosis of UCassociated dysplasia is associated with a small risk of $\mathrm{CRC}$, a finding that deviates significantly from a number of previous studies. While the risk of developing invasive cancer was between 2-3\% over a follow up period of 4.9 (IQR: 2.5-8.9; mean 22.1+/30.9) months, all malignancies were histopathologically staged as N0-stage . The findings will require validation by future studies, but the data may help to inform the decisionmaking process for patients with asymptomatic UC who are considering intensive surveillance rather than surgery after a diagnosis of dysplasia. 
Figure 1 - Time distribution of surgical procedures for patients with UC-associated dysplasia by year through the study period. This figure demonstrates the proportion of the study group who elected to have surgery on a year by year basis.

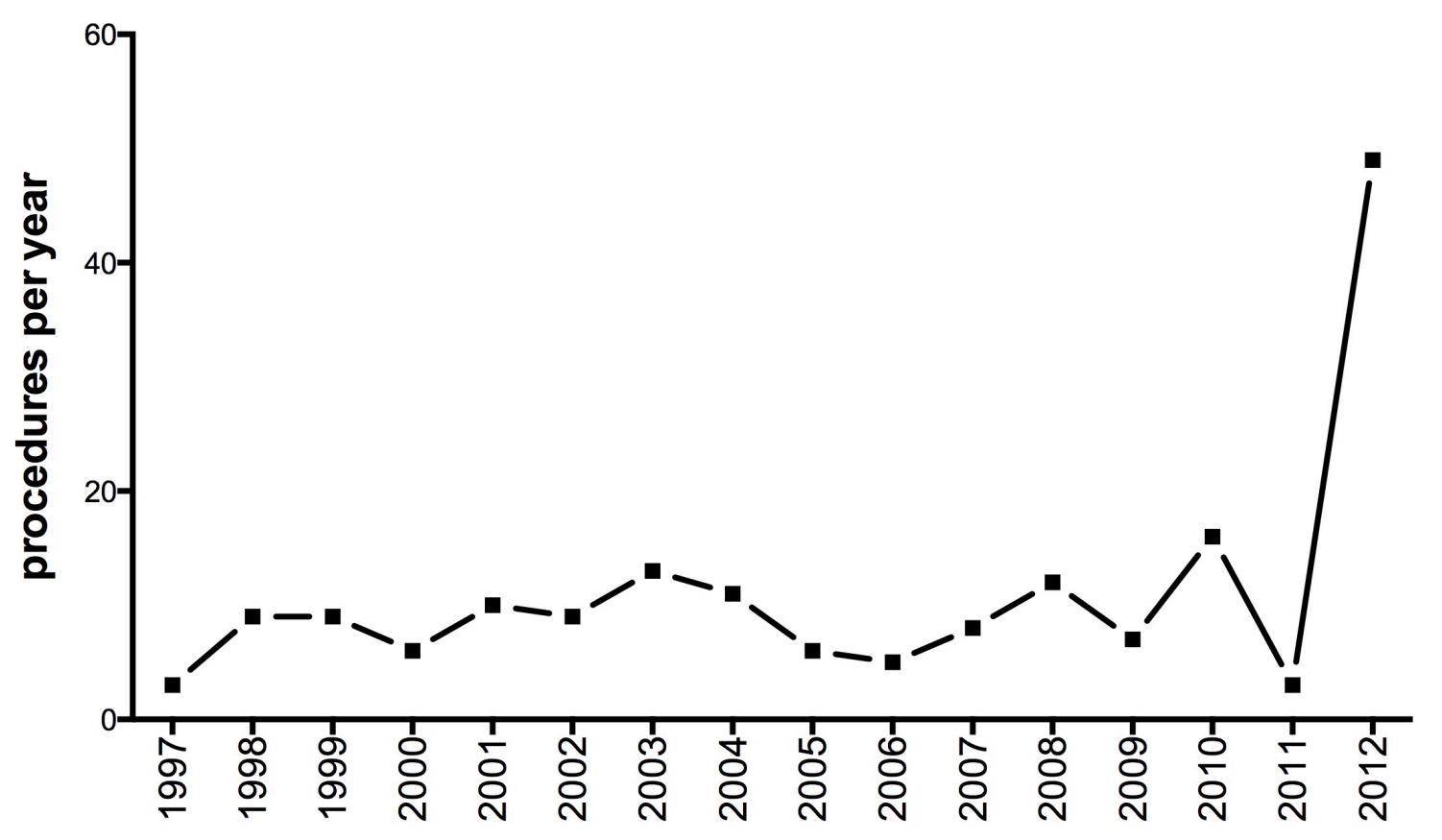


$\underline{\text { Figure 2 }}$ - Flow chart outlining the progression of all 175 patients diagnosed with dysplasia on index endoscopy with histological examination of biopsy material who underwent surgery. All patients initially presented with either indeterminate $(n=77)$ or low-grade ( $\mathrm{n}=98)$ dysplasia, after which some patients were found to have undergone dysplastic progression as indicated by below.

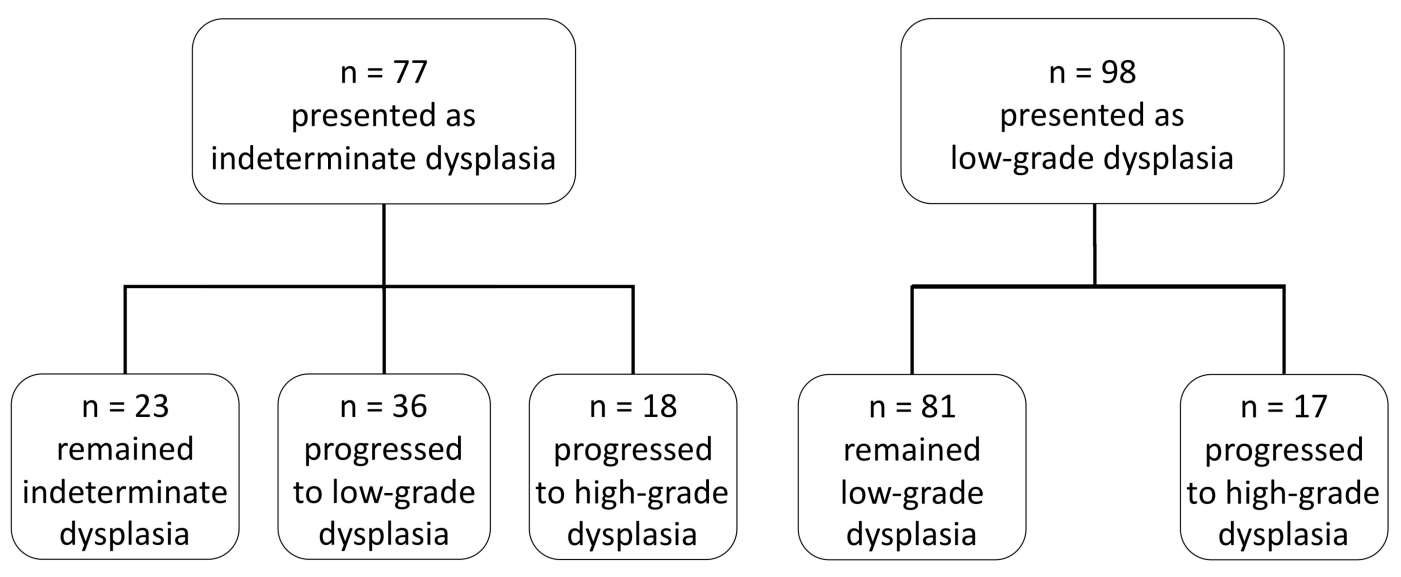


Figure 3 - The interval from diagnosis of indeterminate (ID), low-grade (LGD) or highgrade (HGD) dysplasia to surgery in patients with CRC and in those with dysplasia only confirmed in the postoperative specimen.

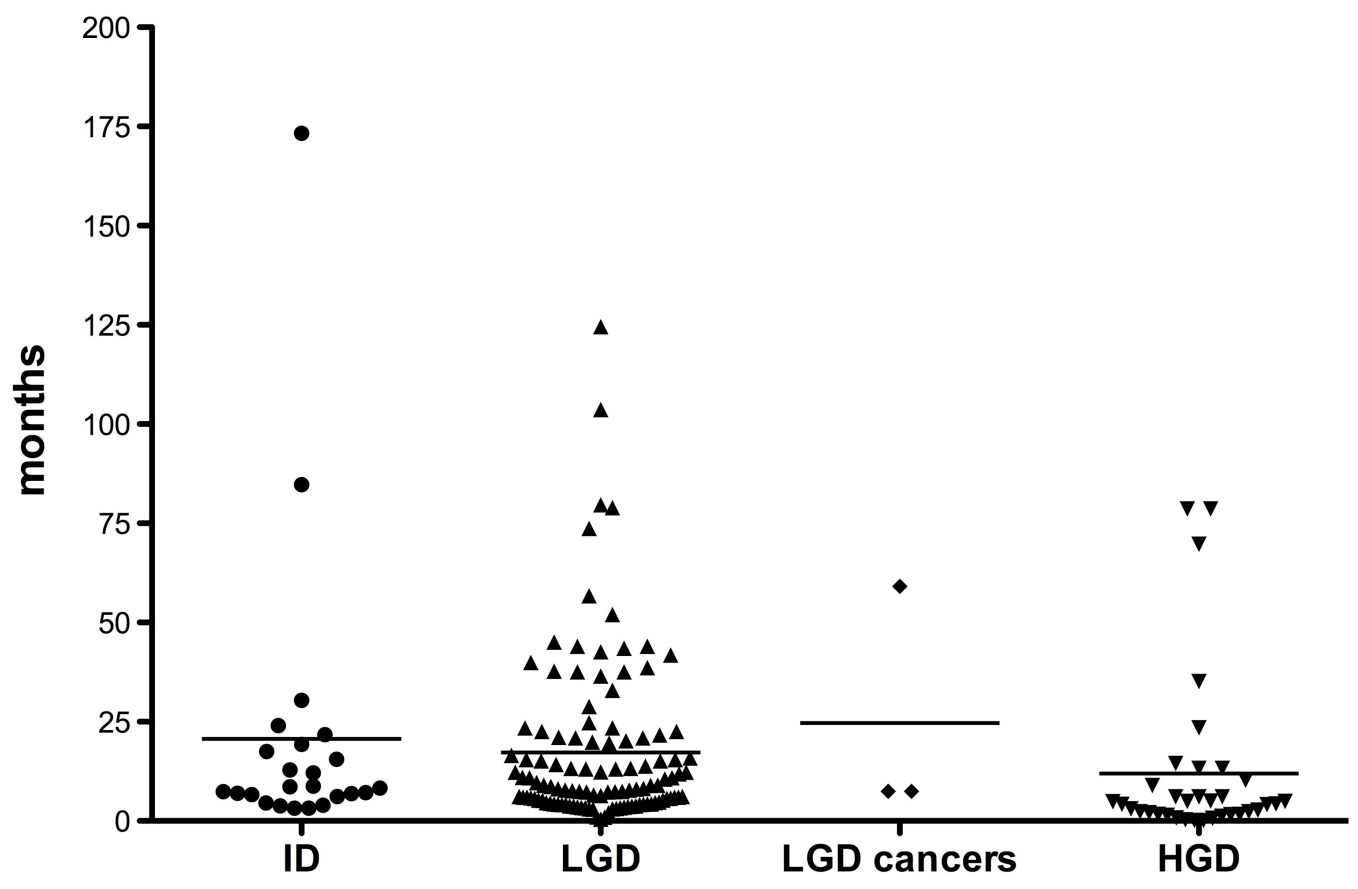


$\underline{\text { Figure } 4}$ - the risk of progression of dysplasia during the study period. Hazard ratios are computed in relation to indeterminate to low-grade dysplastic progression as the denominator.

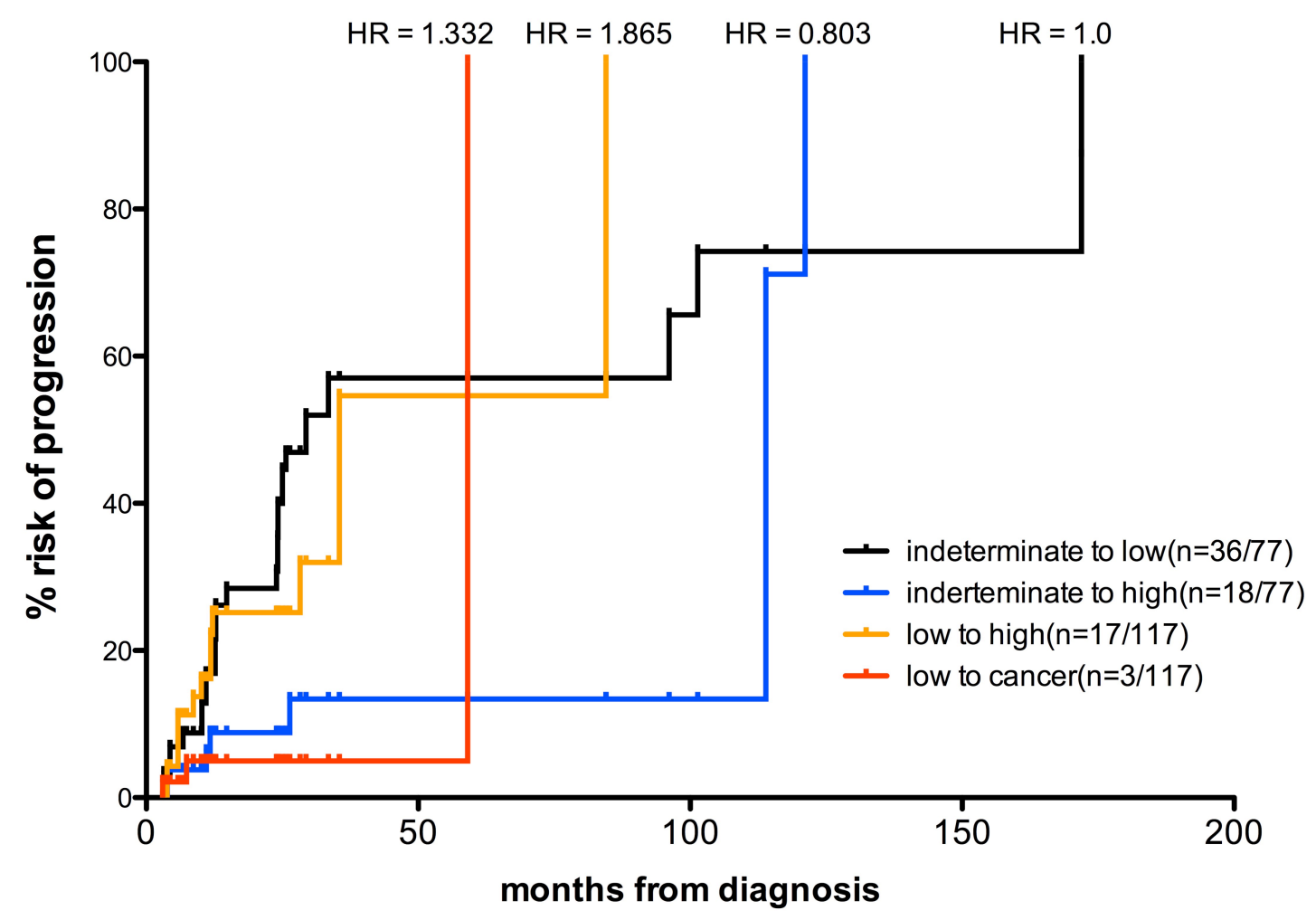




\section{REFERENCES}

1 - Efthymiou M, Taylor AC, Kamm MA. Cancer surveillance strategies in ulcerative colitis: the need for modernization. Inflamm Bowel Dis. 2011 Aug;17(8):1800-13.

2 - Ekbom A, Helmick C, Zack M et al. Ulcerative colitis and colorectal cancer. A population-based study. N Engl J Med. 1990; 323: 1228-1233.

3 - Bernstein $\mathrm{CN}$, Blanchard JF, Kliewer $\mathrm{E}$ et al. Cancer risk in patients with inflammatory bowel disease: a population-based study. Cancer. 2001; 91: 854-862.

4 - van Schaik FD, Offerhaus GJ, Schipper ME et al. Endoscopic and pathological aspects of colitis-associated dysplasia. Nat Rev Gastroenterol Hepatol. 2009 Nov;6(11):671-8.

5 - Farraye FA, Odze RD, Eaden J et al. AGA technical review on the diagnosis and management of colorectal neoplasia in inflammatory bowel disease. Gastroenterology. 2010; 138: 746-774.

6 - Farraye FA, Odze RD, Eaden J et al. AGA medical position statement on the diagnosis and management of colorectal neoplasia in inflammatory bowel disease. Gastroenterology. 2010; 138: 738-745. 
7 - Thomas T, Abrams KA, Robinson RJ et al. Meta-analysis: cancer risk of low-grade dysplasia in chronic ulcerative colitis. Aliment Pharmacol Ther. 2007; 25: 657-668.

8 - Zisman TL, Bronner MP, Rulyak S et al. Prospective study of the progression of lowgrade dysplasia in ulcerative colitis using current cancer surveillance guidelines. Inflamm Bowel Dis. 2012 Dec;18(12):2240-6.

9 - van Schaik FD, ten Kate FJ, Offerhaus GJ et al. Dutch Initiative on Crohn and Colitis. Misclassification of dysplasia in patients with inflammatory bowel disease: consequences for progression rates to advanced neoplasia. Inflamm Bowel Dis. 2011 May;17(5):110816.

10 - Rozen P, Baratz M, Fefer F et al. Low incidence of significant dysplasia in a successful endoscopic surveillance program of patients with ulcerative colitis. Gastroenterology. 1995 May;108(5):1361-70.

11 - Woolrich AJ, DaSilva MD, Korelitz BI. Surveillance in the routine management of ulcerative colitis: the predictive value of low-grade dysplasia. Gastroenterology. 1992 Aug;103(2):431-8.

12 - Jess T, Loftus EV Jr, Velayos FS et al. Incidence and prognosis of colorectal dysplasia in inflammatory bowel disease: a population-based study from Olmsted County, Minnesota. Inflamm Bowel Dis. 2006 Aug;12(8):669-76. 
13 - Cairns SR, Scholefield JH, Steele RJ et al. British Society of Gastroenterology; Association of Coloproctology for Great Britain and Ireland. Guidelines for colorectal cancer screening and surveillance in moderate and high risk groups (update from 2002). Gut. 2010 May;59(5):666-89.

14 - Navaneethan U, Jegadeesan R, Gutierrez NG, Venkatesh PG, Hammel JP, Shen B, Kiran RP. Progression of low-grade dysplasia to advanced neoplasia based on the location and morphology of dysplasia in ulcerative colitis patients with extensive colitis under colonoscopic surveillance. J Crohns Colitis. 2013 Dec 15;7(12):e684-91.

15 - Siegel CA, Schwartz LM, Woloshin S, Cole EB, Rubin DT, Vay T, Baars J, Sands BE. When should ulcerative colitis patients undergo colectomy for dysplasia? Mismatch between patient preferences and physician recommendations. Inflamm Bowel Dis. 2010 Oct;16(10):1658-62.

16 - Kekilli M, Dagli U, Kalkan IH, Tunc B, Disibeyaz S, Ulker A, Sahin B. Low incidence of colorectal dysplasia and cancer among patients with ulcerative colitis: a Turkish referral centre study. Scand J Gastroenterol. 2010 Apr;45(4):434-9.

17 -Pekow JR, Hetzel JT, Rothe JA, Hanauer SB, Turner JR, Hart J, Noffsinger A, Huo D, Rubin DT. Outcome after surveillance of low-grade and indefinite dysplasia in patients with ulcerative colitis. Inflamm Bowel Dis. 2010 Aug;16(8):1352-6. 
18 - Rispo A, Castiglione F, Staibano S, Esposito D, Maione F, Siano M, Salvatori F, Masone S, Persico M, De Palma GD. Diagnostic accuracy of confocal laser endomicroscopy in diagnosing dysplasia in patients affected by long-standing ulcerative colitis. World J Gastrointest Endosc. 2012 Sep 16;4(9):414-20.

19 - Efthymiou M, Allen PB, Taylor AC, Desmond PV, Jayasakera C, De Cruz P, Kamm MA. Chromoendoscopy versus narrow band imaging for colonic surveillance in inflammatory bowel disease. Inflamm Bowel Dis. 2013 Sep;19(10):2132-8.

20 - Gounaris E, Martin J, Ishihara Y, Khan MW, Lee G, Sinh P, Chen EZ, Angarone M, Weissleder R, Khazaie K, Barrett TA. Fluorescence endoscopy of cathepsin activity discriminates dysplasia from colitis. Inflamm Bowel Dis. 2013 Jun;19(7):1339-45.

21 - Nishiyama S, Oka S, Tanaka S, Hayashi N, Hayashi R, Nagai K, Ueno Y, Shimamoto F, Arihiro K, Chayama K. Is it possible to discriminate between neoplastic and nonneoplastic lesions in ulcerative colitis by magnifying colonoscopy? Inflamm Bowel Dis. 2014 Mar;20(3):508-13. 